\title{
Traumatic Stress or ADHD? Making a Case for Trauma Informed Care in Pediatric Practice
}

\author{
Prahbhjot Malhi ${ }^{1} \cdot$ Bhavneet Bharti $^{1}$
}

Received: 29 August 2020 / Accepted: 14 October 2020 / Published online: 23 October 2020

(C) Dr. K C Chaudhuri Foundation 2020

To the Editor: It has long been recognized that adverse childhood experiences (ACEs) (such as childhood exposure to emotional, physical and sexual abuse; emotional and physical neglect) are complex etiological stressors associated with neurobiological alterations that increase the vulnerability of the child to medical and mental health disorders throughout the life span [1]. Evidence indicates that children may respond to traumatic experiences by behaviors that may mimic the symptoms of attention-deficit/hyperactivity disorder (ADHD) or alternatively trauma may exacerbate preexisting ADHD in children [2].

A 5-y-old girl, student of kindergarten, presented with a 3 mo history of emotional dysregulation, hyperactivity, aggression, demanding and stubborn behavior. There was a significant family history of unemployment, family conflict, punitive disciplining, and depressive symptoms in the mother. These factors further increased the risk for maltreatment and deteriorating parent-child relationship. A trauma-focused approach to management was implemented in view of the child's young age, sub-threshold presentation of ADHD, and a high ACE score on the ACE-Q child questionnaire [3]. Management focused on creating a safe therapeutic environment along with enhancing parent-child attachment. The mother was encouraged to hug, engage, ask questions, read to, and talk to the child (HEART acronym strategy) [4].

Young children who are exposed to ACEs can experience intrusive thoughts of trauma and be in a constant state of emotional arousal which can resemble the hyperactivity and impulsivity seen in the ADHD disorder. Indeed, exposure to multiple adversities, play a critical role in the onset and pro-

Prahbhjot Malhi

pmalhi18@hotmail.com

1 Department of Pediatrics, Post Graduate Institute of Medical Education and Research, Sector 12, Chandigarh 160012, India gression of ADHD symptoms [5]. Narrowly focusing on the management of presenting symptoms, without addressing the underlying etiologically relevant trauma history can result in an erroneous diagnosis and ineffective interventions $[2,5]$. Significant efforts are needed to raise awareness about early family and community risks and their impact on brain development. Routine screening for adversity should be incorporated while assessing children who present with challenging behaviors. Pediatricians need to provide trauma-informed care interventions when they encounter childhood experiences. Such a developmentally sensitive framework would widen the boundaries of pediatric practice and make pediatricians more efficacious in enhancing the quality of care and well being of children living in adversity.

\section{Compliance with Ethical Standards}

Conflict of Interest None.

\section{References}

1. Kan K, Gupta R, Davis MM, Heard-Garris N, Garfield C. Adverse experiences and special health care needs among children. Matern Child Health J. 2020;24:552-60.

2. Biederman J, Petty CR, Spencer TJ, et al. Examining the nature of the comorbidity between pediatric attention deficit/hyperactivity disorder and post-traumatic stress disorder. Acta Psychiatr Scand. 2013;128:78-87.

3. Burke Harris N, Renschler T. Center for youth wellness ACEquestionnaire (CYW ACE-Q child). San Francisco: Center for Youth Wellness; 2015.

4. Marsac ML, Kassam-Adams N, Hildenbrand AK, et al. Implementing a trauma-informed approach in pediatric health care networks. JAMA Pediatr. 2016;170:70-7.

5. Brown NM, Brown SN, Briggs RD, Germán M, Belamarich PF, Oyeku SO. Associations between adverse childhood experiences and ADHD diagnosis and severity. Acad Pediatr. 2017;17:349-55.

Publisher's Note Springer Nature remains neutral with regard to jurisdictional claims in published maps and institutional affiliations. 